BMJ Open

Diabetes

Research

\& Care

\section{Combined and sequential non-invasive approach to diagnosing non-alcoholic steatohepatitis in patients with non- alcoholic fatty liver disease and persistently normal alanine aminotransferase levels}

To cite: Zheng Kl, Liu W-Y, Pan X-Y, et al. Combined and sequential non-invasive approach to diagnosing nonalcoholic steatohepatitis in patients with non-alcoholic fatty liver disease and persistently normal alanine aminotransferase levels. BMJ Open Diab Res Care 2020;8:e001174. doi:10.1136/ bmjdrc-2020-001174

- Additional material is published online only. To view please visit the journal online (http://dx.doi.org/10.1136/ bmjdrc-2020-001174).

Received 4 January 2020 Revised 4 February 2020 Accepted 9 February 2020

Check for updates

C A Author(s) (or their employer(s)) 2020. Re-use permitted under CC BY-NC. No commercial re-use. See rights and permissions. Published by BMJ.

For numbered affiliations see end of article.

Correspondence to Professor Ming-Hua Zheng; zhengmh@wmu.edu.cn and Professor Fengmin Lu; lu.fengmin@bjmu.edu.cn

\section{ABSTRACT}

Background and aim Imaging-confirmed nonalcoholic fatty liver disease (NAFLD) with normal alanine aminotransferase (nALT) levels is infrequently the subject for further evaluation. Early diagnosis of non-alcoholic steatohepatitis (NASH) is needed to prevent disease progression. Thus, we tested the clinical utility of serum Golgi protein 73 (GP73) levels and developed a new noninvasive score to diagnose NASH in patients with biopsyconfirmed NAFLD and persistent nALT levels.

Methods Serum GP73 and cytokeratin-18 M30 fragments (CK18-M30) levels were measured in 345 patients with biopsy-proven NAFLD. We developed a new score, named G-NASH model (by incorporating serum GP73), and combined it with serum CK18-M30 measurement in a sequential non-invasive approach to accurately identify NASH among patients with NAFLD and persistent nALT levels.

Results 105 (30.4\%) patients had persistent nALT, 53 of whom had histologically confirmed NASH. Both serum GP73 and CK18-M30 levels alone had poor diagnostic accuracy in identifying NASH $(55.2 \%$ and $51.6 \%$, respectively) in these patients. Conversely, G-NASH model performed better than other established non-invasive scoring systems, and by using our proposed sequential non-invasive approach $82.9 \%$ of patients with NASH were correctly identified.

Conclusions NASH is highly prevalent in patients with NAFLD with persistent nALT levels. The G-NASH model accurately identifies NASH in this patient group.

\section{INTRODUCTION}

With an estimated global prevalence of nearly $25 \%$, non-alcoholic fatty liver disease (NAFLD) is predicted to cause considerable health, social and economic burden in the next few years. ${ }^{1}$ In patients with NAFLD, abnormal serum alanine aminotransferase (ALT) levels are commonly used (both in China and in other countries)

\section{Significance of this study}

What is already known about this subject?

- Liver disease severity is often not investigated further in patients with imaging-confirmed nonalcoholic fatty liver disease (NAFLD) who have normal alanine aminotransferase (ALT) levels.

- Diagnosing non-alcoholic steatohepatitis (NASH) non-invasively remains a big challenge.

What are the new findings?

- We have tested whether serum Golgi protein 73 levels can predict NASH in patients with biopsyconfirmed NAFLD and normal ALT levels.

> Our newly developed non-invasive score (G-NASH) performed best in accurately identifying NASH when compared with other widely used non-invasive scoring systems.

How might these results change the focus of research or clinical practice?

- By using our proposed combined and sequential non-invasive approach, $82.9 \%$ of patients with NASH were correctly identified.

- This is a step toward identifying those patients with NASH with normal ALT as well as reducing the number of patients who may be subject to unnecessary liver biopsy.

as a surrogate marker of a more severe liver condition, such as non-alcoholic steatohepatitis (NASH), and when NASH occurs with advanced fibrosis or cirrhosis there is also a marked increase in risk of hepatocellular carcinoma or end-stage liver disease. ${ }^{2}$ As a consequence, patients with NAFLD with persistently normal ALT (nALT) levels are often neglected from further assessment of the severity of their 
liver disease. ${ }^{3}$ This is clinically important because there is little evidence that the risk of developing liver-related complications or extrahepatic diseases in this group of patients is any different from that of their counterparts with abnormal ALT levels. ${ }^{4}$ Moreover, the prevalence of patients with NAFLD who have normal serum ALT levels is high. For example, the prevalence of patients with serum ALT levels within the upper limit of normal (ULN) was reported to be over $90 \%$ of US adult individuals with NAFLD, according to the National Health and Nutrition Examination Survey III. ${ }^{5}$

To date, an understanding of the liver disease severity and the characteristics of patients with NAFLD who have nALT levels is limited. While most studies have included patients with NAFLD and serum levels of ALT above the ULN (abnALT), patients with persistent nALT have been included only in a few studies of NAFLD. ${ }^{6}$ Although serum ALT levels are a poor predictor of the histologic severity of NAFLD,${ }^{27}$ serum ALT levels remain to date the most widely used laboratory parameter, both in China and in other countries, for referral to liver specialist(s) in those patients with suspected more severe liver disease. ${ }^{3}$

Increased levels of serum cytokeratin-18 (CK18) have shown promising results in diagnosing NASH in some studies, but it is uncertain whether serum CK18 levels may also be useful in diagnosing NASH in patients with persistent nALT. ${ }^{8}$

Golgi membrane glycoprotein 73 (GP73), also known as Golgi phosphoprotein 2, is a type II Golgi-localized integral membrane protein found in the blood when it is cleaved by the proprotein convertase furin. In a previous study, we found that elevated serum GP73 levels were a reliable marker of liver fibrosis and cirrhosis. ${ }^{9}$ Elevated serum GP73 levels were also found to be useful in assessing liver inflammation in chronic viral hepatitis B. ${ }^{10}$ Also, Lu and Zhang ${ }^{11}$ confirmed the usefulness of GP73 levels in NAFLD-related cirrhosis. Because GP73 is released into the serum as a result of hepatic injury and is associated with severity of liver inflammation in chronic liver diseases, we believe that its diagnostic utility in patients with NASH with persistent nALT should also be examined.

Therefore, the aim of our study was to test, in patients with biopsy-proven NAFLD, the diagnostic performance of a combined and sequential strategy, using serum cytokeratin-18 M30 fragments (CK18-M30) and GP73 levels, in predicting NASH in patients with persistent nALT levels.

\section{METHODS}

Patients

This is an observational study of the well-characterized Prospective Epidemic Research Specifically Of NASH (PERSONS) cohort. $^{12}$ Patients enrolled in this study fulfilled the following criteria: (1) age between 18-75 years; (2) presence of hepatic steatosis on liver ultrasound or other imaging techniques, and then confirmed by liver biopsy, in the absence of other competing causes of chronic liver diseases (as specified in the Results section); and (3) provided informed written consent prior to their inclusion in the study. Persistent nALT status was defined as a serum ALT level below the ULN (ie, $<40 \mathrm{U} / \mathrm{L}$ for both sexes, according to our local laboratory reference) for at least 3 months prior to study entry.

\section{Laboratory, anthropometric and clinical data}

As previously reported, anthropometric measurements were recorded, and all blood samples were obtained on the same day of liver biopsy, after at least an 8-hour fast, and routine biochemical tests performed in our central laboratory. ${ }^{13}$ Serum insulin and total thyroxine concentrations were measured using commercially available radioimmunoassay kits. Body mass index (BMI) was calculated as weight $(\mathrm{kg})$ divided by squared height $(\mathrm{m})$.

The presence of diabetes was defined as a selfreported, physician-established diagnosis of diabetes, current use of any hypoglycemic drugs, a fasting glucose level $\geq 7.0 \mathrm{mmol} / \mathrm{L}(\geq 126 \mathrm{mg} / \mathrm{dL})$ or a hemoglobin Alc level $\geq 6.5 \%(\geq 48 \mathrm{mmol} / \mathrm{mol})$. Diagnosis of metabolic syndrome was based on the presence of at least three of the following metabolic abnormalities: (1) waist circumference $>90 \mathrm{~cm}$ in men and $>80 \mathrm{~cm}$ in women; (2) triglycerides $\geq 1.70 \mathrm{mmol} / \mathrm{L}$; (3) high-density lipoprotein $<1.0 \mathrm{mmol} / \mathrm{L}$ in men and $<1.3 \mathrm{mmol} / \mathrm{L}$ in women; (4) blood pressure $\geq 130 / 85 \mathrm{~mm} \mathrm{Hg}$ or on drug treatment; and (5) fasting glucose $\geq 5.6 \mathrm{mmol} / \mathrm{L}$. Hypertension was defined as blood pressure $\geq 140 / 90 \mathrm{~mm} \mathrm{Hg}$ or use of any antihypertensive agents. Hyperuricemia was defined as a serum uric acid level $>428 \mu \mathrm{mol} / \mathrm{L}$ or use of allopurinol. Homeostasis model assessment of insulin resistance was calculated by published formula. ${ }^{14}$ Obesity in Asian individuals was defined as BMI $\geq 25 \mathrm{~kg} / \mathrm{m}^{2}$. ${ }^{15}$

\section{Clinical scoring systems for predicting NASH}

The currently proposed scoring systems for predicting NASH include the NAFIC score, the Palekar $e t a l$ s risk factors panel, the Cao et als score system, and the Nice model. $^{16-19}$

\section{Measurements of serum GP73 and CK18}

Serum GP73 and CK18-M30 concentrations were measured by two commercially ELISA kits that were provided by Hotgen Biotech (Beijing, China) and Herui Biomed Company (Suzhou, China), respectively. Serum samples were stored at $-80^{\circ} \mathrm{C}$ prior to testing. Both tests had coefficients of variation $<15 \%$ and were performed by a technician, who was blinded to participants' clinical details. The upper normal limits of serum GP73 and CK18-M30 levels in healthy population, provided by the manufacturer of commercial kits, were $53 \mathrm{ng} / \mathrm{mL}$ and $200 \mathrm{U} / \mathrm{L}$, respectively.

\section{Liver biopsy}

In brief, liver biopsy was performed and histology was assessed, separately, by an expert liver pathologist (X-DW) ${ }^{20}$ who was blinded to clinical and biochemical data of participants, according to the NASH Clinical Research Network scoring system. ${ }^{21}$ In this study NASH was defined as the presence of NAFLD Activity Score $\geq 4$, 
with at least one point for each of the three individual features of NAFLD (ie, steatosis, lobular inflammation, and ballooning), in the absence of other known causes of chronic liver disease. Liver fibrosis was scored from 0 to 4 according to Brunt's criteria. ${ }^{22}$

\section{Statistical analysis}

Continuous variables were expressed as mean $\pm \mathrm{SD}$ and compared using either the Student's t-test for normally distributed variables or the Mann-Whitney test for nonnormally distributed variables. Difference between categorical variables was examined with the $\chi^{2}$ test or the Fisher's exact test as appropriate. A $p$ value $<0.05$ was considered statistically significant. Data management and analysis were performed using R software (V.3.5.2; R Foundation for Statistical Computing, Vienna, Austria).

Clinically relevant variables were preselected and subjected to univariable regression analysis. Since nALT levels may differ between populations, ${ }^{23}$ exploratory analysis was performed with a lower cut-off value of serum ALT levels ( $\leq 35 \mathrm{U} / \mathrm{L}$ for men and $\leq 23 \mathrm{U} / \mathrm{L}$ for women), referring to our previous study in the Chinese Han population. ${ }^{24} \mathrm{~A}$ multivariable regression model was also determined by (1) backward stepwise logistic regression (SLR) and (2) best-subset regression (BSR). Variables in univariable regression analysis with a $\mathrm{p}$ value $<0.10$ were entered into SLR, and the model was determined with the lowest value of Akaike's information criterion. Variables in univariable analysis with a $p$ value $<0.30$ were selected to form a whole set for exhaustive search by BSR.

Subsequently, a receiver operating characteristic curve (ROC) was performed to evaluate the aforementioned models. The area under the receiver operating characteristic curve (AUROC), sensitivity, specificity, positive predictive value (PPV) and negative predictive value (NPV) were calculated to assess the diagnostic accuracy of models or predictors. Sensitivity and specificity were given under the maximal Youden's Index (sensitivity+specificity-1).

To further determine whether incorporation of the models or predictors could improve outcome(s) of patients in clinical practice, a decision curve analysis (DCA) was undertaken using the $r m d a$ R package. DCA uses net benefit (NB) to assess the performance of predictive risk models. The standardized net benefit ( $\mathrm{sNB}$ ) was a convenient form of NB to be interpreted, which was defined as NB divided by prevalence of disease. The cost to benefit ratio (CBR) was calculated as the odds of $\mathrm{P}_{\mathrm{t}}\left(\mathrm{CBR}=\mathrm{P}_{\mathrm{t}} /\left(1-\mathrm{P}_{\mathrm{t}}\right)\right){ }^{25}$

\section{RESULTS}

\section{Baseline characteristics}

Between December 2016 and September 2018, a total of 588 individuals with imaging-defined fatty liver were screened. Among these, 244 were excluded for the following reasons: 79 had alcoholic fatty liver, 75 had steatosis $<5 \%$ on liver histology, 71 had viral hepatitis, 7 had autoimmune hepatitis, 3 had drug-induced hepatitis and 8 had missing data for serum GP73 levels. As a result, 345 patients with biopsyconfirmed NAFLD were included in statistical analyses and were then divided into two subgroups according to their serum ALT levels. The flow chart for patient selection process is shown in figure 1 .

Table 1 shows the main clinical and biochemical characteristics of patients with biopsy-proven NAFLD stratified by

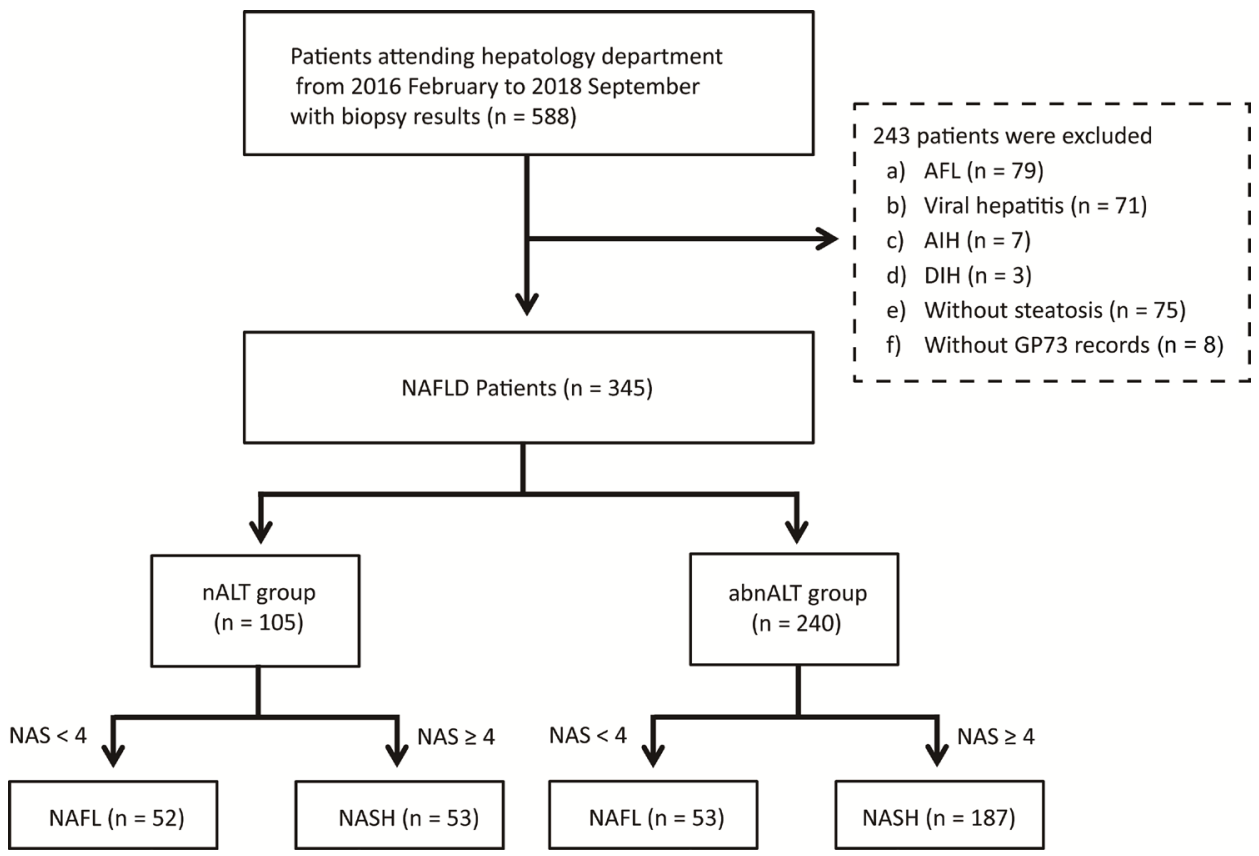

Figure 1 Flow diagram of the study. abnALT, alanine aminotransferase above the upper limit of normal; AFL, alcoholic fatty liver; AIH, autoimmune hepatitis; DIH, drug-induced hepatitis; GP73, Golgi protein 73; NAFL, non-alcoholic fatty liver; NAFLD, non-alcoholic fatty liver disease; nALT, normal alanine aminotransferase; NAS, NAFLD Activity Score; NASH, non-alcoholic steatohepatitis. 
Table 1 Baseline characteristics of patients with biopsy-proven NAFLD

\begin{tabular}{|c|c|c|c|c|}
\hline & & Serum ALT leve & & \\
\hline & $\begin{array}{l}\text { Overall } \\
\text { (N=345) }\end{array}$ & $\begin{array}{l}\text { abnALT group } \\
(n=240)\end{array}$ & $\begin{array}{l}\text { nALT group } \\
(n=105)\end{array}$ & $P$ value \\
\hline Female sex, n (\%) & $83(24.1)$ & $45(18.8)$ & $38(36.2)$ & 0.001 \\
\hline Age, years & $40.2 \pm 12.5$ & $37.8 \pm 12.1$ & $45.5 \pm 11.6$ & $<0.001$ \\
\hline WCC, $\times 10^{9} / \mathrm{L}$ & $6.22 \pm 1.50$ & $6.41 \pm 1.60$ & $5.80 \pm 1.12$ & $<0.001$ \\
\hline $\mathrm{PLT}, \times 10^{9} / \mathrm{L}$ & $245.48 \pm 57.72$ & $243.62 \pm 58.69$ & $249.72 \pm 55.50$ & 0.367 \\
\hline MPV, fL & $10.92 \pm 0.99$ & $10.95 \pm 1.02$ & $10.87 \pm 0.92$ & 0.506 \\
\hline SDPV, fL & $13.80 \pm 2.77$ & $13.94 \pm 3.02$ & $13.48 \pm 2.09$ & 0.160 \\
\hline Albumin, g/L & $46.76 \pm 3.74$ & $47.34 \pm 3.19$ & $45.43 \pm 4.50$ & $<0.001$ \\
\hline ALT, U/L & $82.47 \pm 77.04$ & $106.43 \pm 81.38$ & $27.70 \pm 7.77$ & $<0.001$ \\
\hline Fasting glucose, $\mathrm{mmol} / \mathrm{L}$ & $5.60 \pm 1.42$ & $5.47 \pm 1.24$ & $5.91 \pm 1.74$ & 0.007 \\
\hline $\mathrm{BUN}, \mathrm{mmol} / \mathrm{L}$ & $4.83 \pm 1.28$ & $4.80 \pm 1.25$ & $4.90 \pm 1.34$ & 0.505 \\
\hline Creatinine, $\mu \mathrm{mol} / \mathrm{L}$ & $67.94 \pm 14.49$ & $68.72 \pm 14.12$ & $66.17 \pm 15.22$ & 0.133 \\
\hline Uric acid, $\mu \mathrm{mol} / \mathrm{L}$ & $403.35 \pm 105.20$ & $420.57 \pm 106.75$ & $364.00 \pm 90.37$ & $<0.001$ \\
\hline Total cholesterol, $\mathrm{mmol} / \mathrm{L}$ & $5.05 \pm 1.11$ & $5.14 \pm 1.09$ & $4.84 \pm 1.15$ & 0.023 \\
\hline Triglycerides, $\mathrm{mmol} / \mathrm{L}$ & $2.30 \pm 1.41$ & $2.31 \pm 1.28$ & $2.27 \pm 1.69$ & 0.774 \\
\hline $\mathrm{HDL}$ cholesterol, $\mathrm{mmol} / \mathrm{L}$ & $1.01 \pm 0.23$ & $1.00 \pm 0.23$ & $1.04 \pm 0.23$ & 0.232 \\
\hline LDL cholesterol, mmol/L & $3.08 \pm 0.92$ & $3.18 \pm 0.89$ & $2.86 \pm 0.95$ & 0.002 \\
\hline GP73, ng/mL & $80.96 \pm 40.05$ & $85.55 \pm 43.02$ & $70.45 \pm 29.88$ & 0.001 \\
\hline AFP, ng/mL & $2.99 \pm 1.42$ & $3.01 \pm 1.34$ & $2.94 \pm 1.60$ & 0.674 \\
\hline Ferritin, $\mu \mathrm{g} / \mathrm{L}$ & $347.71 \pm 242.07$ & $393.36 \pm 252.50$ & $243.25 \pm 178.13$ & $<0.001$ \\
\hline Type III procollagen, ng/mL & $21.38 \pm 19.74$ & $22.80 \pm 23.42$ & $18.21 \pm 4.77$ & 0.049 \\
\hline Type IV collagen, ng/mL & $20.57 \pm 9.57$ & $21.73 \pm 10.87$ & $18.00 \pm 4.80$ & 0.001 \\
\hline Hyaluronic acid, ng/mL & $48.26 \pm 39.89$ & $48.49 \pm 46.30$ & $47.74 \pm 19.22$ & 0.874 \\
\hline Comorbid diseases, n (\%) & & & & \\
\hline Diabetes & $104(30.1)$ & $66(27.5)$ & $38(36.2)$ & 0.136 \\
\hline Metabolic syndrome & $199(60.3)$ & $144(62.9)$ & $55(54.5)$ & 0.187 \\
\hline Hypertension & $111(32.2)$ & $74(30.8)$ & $37(35.2)$ & 0.496 \\
\hline Dyslipidemia & $313(91.5)$ & $221(92.9)$ & $92(88.5)$ & 0.258 \\
\hline Hyperuricemia & $131(38.0)$ & $107(44.6)$ & $24(22.9)$ & $<0.001$ \\
\hline $\begin{array}{l}\text { Obesity } \\
\text { BMl } 25-29.9 \mathrm{~kg} / \mathrm{m}^{2}\end{array}$ & $190(55.1)$ & $143(59.6)$ & $47(44.8)$ & 0.015 \\
\hline $\begin{array}{l}\text { Obesity } \\
\text { BMI } \geq 30 \mathrm{~kg} / \mathrm{m}^{2}\end{array}$ & $48(14.0)$ & $37(15.5)$ & $11(10.5)$ & 0.287 \\
\hline Anthropometry & & & & \\
\hline Waist circumference, $\mathrm{cm}$ & $92.25 \pm 8.70$ & $93.30 \pm 8.44$ & $89.87 \pm 8.85$ & 0.001 \\
\hline Hip circumference, $\mathrm{cm}$ & $99.69 \pm 7.46$ & $100.63 \pm 7.27$ & $97.57 \pm 7.50$ & 0.001 \\
\hline $\mathrm{BMI}, \mathrm{kg} / \mathrm{m}^{2}$ & $26.93 \pm 4.20$ & $27.31 \pm 4.49$ & $26.05 \pm 3.33$ & 0.010 \\
\hline
\end{tabular}




\begin{tabular}{|c|c|c|c|c|}
\hline & & Serum ALT leve & & \\
\hline & $\begin{array}{l}\text { Overall } \\
(\mathrm{N}=345)\end{array}$ & $\begin{array}{l}\text { abnALT group } \\
(n=240)\end{array}$ & $\begin{array}{l}\text { nALT group } \\
(n=105)\end{array}$ & $P$ value \\
\hline Liver histology & & & & \\
\hline NASH, n (\%) & $240(69.6)$ & $187(77.9)$ & $53(50.5)$ & $<0.001$ \\
\hline Steatosis, $\mathrm{n}(\%)$ & & & & $<0.001$ \\
\hline 1 & $142(41.2)$ & $67(27.9)$ & $75(71.4)$ & \\
\hline 2 & $91(26.4)$ & $73(30.4)$ & $18(17.1)$ & \\
\hline 3 & $112(32.5)$ & $100(41.7)$ & $12(11.4)$ & \\
\hline Hepatocyte ballooning, $\mathrm{n}(\%)$ & & & & 0.272 \\
\hline 0 & $13(3.8)$ & $8(3.3)$ & $5(4.8)$ & \\
\hline 1 & $182(52.8)$ & $121(50.4)$ & $61(58.1)$ & \\
\hline 2 & $150(43.5)$ & $111(46.2)$ & $39(37.1)$ & \\
\hline Lobular inflammation, n (\%) & & & & 0.004 \\
\hline 0 & $21(6.1)$ & $11(4.6)$ & $10(9.5)$ & \\
\hline 1 & 249 (72.2) & $165(68.8)$ & $84(80.0)$ & \\
\hline 2 & $70(20.3)$ & $60(25.0)$ & $10(9.5)$ & \\
\hline 3 & $5(1.4)$ & $4(1.7)$ & $1(1.0)$ & \\
\hline Fibrosis, n (\%) & & & & 0.059 \\
\hline 0 & $111(32.3)$ & $69(28.9)$ & $42(40.0)$ & \\
\hline 1 & $161(46.8)$ & $124(51.9)$ & 37 (35.2) & \\
\hline 2 & $55(16.0)$ & $34(14.2)$ & $21(20.0)$ & \\
\hline 3 & $16(4.7)$ & $11(4.6)$ & $5(4.8)$ & \\
\hline 4 & $1(0.3)$ & $1(0.4)$ & $0(0.0)$ & \\
\hline
\end{tabular}

Continuous variables are presented as mean \pm SD; categorical variables are presented as $\mathrm{n}(\%)$.

$P$ values $<0.05$ are in bold.

abnALT, ALT above the upper limit of normal; AFP, alpha-fetoprotein; ALP, alkaline phosphatase; ALT, alanine aminotransferase; AST, aspartate aminotransferase; BMI, body mass index; BUN, blood urea nitrogen; CK18-M30, cytokeratin-18 M30 fragments; GGT, gamma-glutamyl transferase; GP73, Golgi protein 73; HbA1c, hemoglobin A1c; HDL, high-density lipoprotein; HOMA-IR, homeostasis model assessment of insulin resistance; LDL, low-density lipoprotein; MPV, mean platelet volume; NAFLD, non-alcoholic fatty liver disease; nALT, normal ALT; NASH, non-alcoholic steatohepatitis; PLT, platelet; RBC, red blood cell; SDPV, SD of platelet volume; WCC, white cell count.

abnALT and persistent nALT (ie, using a cut-off of serum ALT $<40 \mathrm{U} / \mathrm{L}$ for both sexes). There were $105(30.4 \%)$ patients with persistent nALT (67 men, 38 women). The mean ALT levels for the abnALT and persistent nALT groups were $106.4 \pm 81.4 \mathrm{U} / \mathrm{L}$ and $27.7 \pm 7.8 \mathrm{U} / \mathrm{L}$, respectively. Serum levels of aspartate aminotransferase (AST), gamma-glutamyl transferase, uric acid, ferritin, GP73, type III procollagen, type IV collagen and CK18-M30 were significantly lower in the persistent nALT group, while this latter group of patients were more likely to be older, leaner and of female sex, compared with the abnALT group. The presence of NASH was greater in the abnALT group than in the persistent nALT group $(77.9 \%$ vs $50.5 \%, \mathrm{p}<0.001)$. Histologically, the abnALT group had more severe hepatic steatosis and lobular inflammation compared with the persistent nALT group, but with comparable stages of liver fibrosis.

To further examine the clinical and biochemical profile of patients with persistent nALT, we subdivided these patients according to the presence/absence of NASH (table 2). In this subgroup, 53 out of 105 (50.5\%) patients had NASH. Interestingly, almost all clinical and biochemical variables (including also serum CK18-M30) were comparable between the two groups, with the only exception for serum AST and GP73 levels which were significantly higher in the NASH group than in the nonNASH group.

\section{Diagnostic performance of serum GP73 and CK18-M30 in identifying NASH in patients with persistent nALT}

Both concentrations of GP73 and CK18-M30 were increased in patients with NASH, regardless of ALT levels (as summarized in online supplementary table 2). Table 3 shows the ability to discriminate NASH from simple steatosis with either serum GP73 or CK18-M30 levels alone in the subgroup of patients with NAFLD with persistent nALT (defined as a serum ALT level $\leq 40 \mathrm{U} / \mathrm{L}$ ). Fifty-eight (55.2\%) patients were accurately identified with NASH using serum GP73 alone, whereas 49 (51.6\%) patients were accurately identified using serum CK18M30 alone. Therefore, the diagnostic accuracies of GP73 or CK18-M30 alone in identifying NASH were low and essentially comparable $(p=0.707)$. Moreover, as also shown in the table, serum GP73 had higher sensitivity and NPV, but lower specificity and PPV, than serum CK18M30. Similar results were found when we used a lower 
Table 2 Baseline characteristics of patients with persistent nALT stratified by NASH status

\begin{tabular}{|c|c|c|c|c|}
\hline & Overall $(n=105)$ & Non-NASH $(n=52)$ & NASH $(n=53)$ & $P$ value \\
\hline \multicolumn{5}{|l|}{ Clinical parameters } \\
\hline Female sex, n (\%) & $38(36.2)$ & $16(30.8)$ & $22(41.5)$ & 0.346 \\
\hline Age, years & $45.5 \pm 11.6$ & $46.2 \pm 9.5$ & $44.9 \pm 13.4$ & 0.571 \\
\hline WCC, $\times 10^{9} / \mathrm{L}$ & $5.80 \pm 1.12$ & $5.76 \pm 1.17$ & $5.84 \pm 1.09$ & 0.735 \\
\hline RBC, $\times 10^{12} / \mathrm{L}$ & $4.78 \pm 0.44$ & $4.80 \pm 0.46$ & $4.76 \pm 0.41$ & 0.626 \\
\hline $\mathrm{PLT}, \times 10^{9} / \mathrm{L}$ & $249.72 \pm 55.50$ & $246.44 \pm 48.22$ & $252.94 \pm 62.13$ & 0.551 \\
\hline MPV, fL & $10.87 \pm 0.92$ & $10.99 \pm 0.79$ & $10.76 \pm 1.02$ & 0.197 \\
\hline SDPV, fL & $13.48 \pm 2.09$ & $13.88 \pm 1.95$ & $13.09 \pm 2.18$ & 0.055 \\
\hline Albumin, g/L & $45.43 \pm 4.50$ & $45.27 \pm 3.10$ & $45.59 \pm 5.58$ & 0.715 \\
\hline ALT, U/L & $27.70 \pm 7.77$ & $26.88 \pm 8.07$ & $28.49 \pm 7.45$ & 0.292 \\
\hline AST, U/L & $25.77 \pm 6.75$ & $24.02 \pm 3.88$ & $27.49 \pm 8.39$ & 0.008 \\
\hline ALP, U/L & $83.96 \pm 45.57$ & $81.06 \pm 24.40$ & $86.81 \pm 59.60$ & 0.520 \\
\hline GGT, U/L & $43.98 \pm 34.37$ & $41.08 \pm 27.35$ & $46.83 \pm 40.15$ & 0.394 \\
\hline Fasting glucose, $\mathrm{mmol} / \mathrm{L}$ & $5.91 \pm 1.74$ & $5.61 \pm 1.36$ & $6.21 \pm 2.01$ & 0.080 \\
\hline $\mathrm{BUN}, \mathrm{mmol} / \mathrm{L}$ & $4.90 \pm 1.34$ & $4.82 \pm 1.09$ & $4.98 \pm 1.55$ & 0.539 \\
\hline Creatinine, $\mu \mathrm{mol} / \mathrm{L}$ & $66.17 \pm 15.22$ & $68.25 \pm 13.77$ & $64.13 \pm 16.39$ & 0.167 \\
\hline Uric acid, $\mu \mathrm{mol} / \mathrm{L}$ & $364.00 \pm 90.37$ & $368.27 \pm 89.92$ & $359.81 \pm 91.48$ & 0.634 \\
\hline Total cholesterol, $\mathrm{mmol} / \mathrm{L}$ & $4.84 \pm 1.15$ & $4.90 \pm 1.03$ & $4.79 \pm 1.26$ & 0.622 \\
\hline Triglycerides, mmol/L & $2.27 \pm 1.69$ & $2.30 \pm 1.92$ & $2.24 \pm 1.43$ & 0.859 \\
\hline HDL cholesterol, mmol/L & $1.04 \pm 0.23$ & $1.01 \pm 0.23$ & $1.06 \pm 0.22$ & 0.243 \\
\hline LDL cholesterol, $\mathrm{mmol} / \mathrm{L}$ & $2.86 \pm 0.95$ & $2.91 \pm 0.90$ & $2.80 \pm 0.99$ & 0.579 \\
\hline GP73, ng/mL & $70.45 \pm 29.88$ & $64.59 \pm 22.07$ & $76.20 \pm 35.21$ & 0.046 \\
\hline CK18-M30, U/L & $102.21 \pm 88.79$ & $95.76 \pm 55.88$ & $108.02 \pm 110.67$ & 0.504 \\
\hline Zinc, $\mu \mathrm{mol} / \mathrm{L}$ & $18.24 \pm 3.98$ & $17.46 \pm 3.61$ & $19.05 \pm 4.24$ & 0.075 \\
\hline Thyroxine, nmol/L & $105.93 \pm 19.24$ & $103.50 \pm 14.29$ & $108.32 \pm 22.99$ & 0.201 \\
\hline $\mathrm{HbA} 1 \mathrm{c}, \%$ & $6.28 \pm 1.53$ & $6.12 \pm 1.60$ & $6.45 \pm 1.46$ & 0.281 \\
\hline Fasting insulin, pmol/L & $132.76 \pm 153.19$ & $125.66 \pm 169.29$ & $139.86 \pm 136.49$ & 0.639 \\
\hline HOMA-IR & $5.99 \pm 9.70$ & $5.48 \pm 10.05$ & $6.51 \pm 9.41$ & 0.592 \\
\hline AFP, $n g / m L$ & $2.94 \pm 1.60$ & $2.96 \pm 1.90$ & $2.91 \pm 1.24$ & 0.877 \\
\hline Ferritin, $\mu \mathrm{g} / \mathrm{L}$ & $243.25 \pm 178.13$ & $214.96 \pm 172.49$ & $272.52 \pm 182.12$ & 0.218 \\
\hline Type III procollagen, ng/mL & $18.21 \pm 4.77$ & $17.30 \pm 4.54$ & $19.13 \pm 4.86$ & 0.050 \\
\hline Type IV collagen, ng/mL & $18.00 \pm 4.80$ & $17.11 \pm 4.31$ & $18.90 \pm 5.14$ & 0.057 \\
\hline Hyaluronic acid, ng/mL & $47.74 \pm 19.22$ & $44.20 \pm 13.93$ & $51.28 \pm 22.94$ & 0.060 \\
\hline \multicolumn{5}{|l|}{ Comorbid diseases, n (\%) } \\
\hline Diabetes & $38(36.2)$ & $16(30.8)$ & $22(41.5)$ & 0.346 \\
\hline Metabolic syndrome & $55(54.5)$ & $25(50.0)$ & $30(58.8)$ & 0.49 \\
\hline Hypertension & 37 (35.2) & 19 (36.5) & $18(34.0)$ & 0.943 \\
\hline Dyslipidemia & $92(88.5)$ & 48 (92.3) & $44(84.6)$ & 0.357 \\
\hline Hyperuricemia & 24 (22.9) & $12(23.1)$ & $12(22.6)$ & 0.999 \\
\hline $\begin{array}{l}\text { Obesity } \\
\text { BMI } 25-29.9 \mathrm{~kg} / \mathrm{m}^{2}\end{array}$ & $47(44.8)$ & $22(42.3)$ & $35(47.2)$ & 0.761 \\
\hline $\begin{array}{l}\text { Obesity } \\
\text { BMI } \geq 30 \mathrm{~kg} / \mathrm{m}^{2}\end{array}$ & $11(10.5)$ & $3(5.8)$ & $8(15.1)$ & 0.214 \\
\hline \multicolumn{5}{|l|}{ Anthropometry } \\
\hline Waist circumference, $\mathrm{cm}$ & $89.87 \pm 8.85$ & $88.57 \pm 9.41$ & $91.12 \pm 8.18$ & 0.148 \\
\hline Hip circumference, $\mathrm{cm}$ & $97.57 \pm 7.50$ & $96.38 \pm 7.20$ & $98.72 \pm 7.67$ & 0.115 \\
\hline $\mathrm{BMI}, \mathrm{kg} / \mathrm{m}^{2}$ & $26.05 \pm 3.33$ & $25.63 \pm 3.26$ & $26.47 \pm 3.38$ & 0.195 \\
\hline
\end{tabular}


Table 2 Continued

\begin{tabular}{|c|c|c|c|c|}
\hline & Overall $(n=105)$ & Non-NASH $(n=52)$ & NASH $(n=53)$ & $P$ value \\
\hline \multicolumn{5}{|l|}{ Liver histology } \\
\hline Steatosis, n (\%) & & & & $<0.001$ \\
\hline 1 & $75(71.4)$ & $51(98.1)$ & $24(45.3)$ & \\
\hline 2 & $18(17.1)$ & $1(1.9)$ & $17(32.1)$ & \\
\hline 3 & $12(11.4)$ & $0(0.0)$ & $12(22.6)$ & \\
\hline Hepatocyte ballooning, n (\%) & & & & $<0.001$ \\
\hline 0 & $5(4.8)$ & $5(9.6)$ & $0(0.0)$ & \\
\hline 1 & $61(58.1)$ & $46(88.5)$ & $15(28.3)$ & \\
\hline 2 & $39(37.1)$ & $1(1.9)$ & $38(71.7)$ & \\
\hline Lobular inflammation, $\mathrm{n}(\%)$ & & & & $<0.001$ \\
\hline 0 & $10(9.5)$ & $10(19.2)$ & $0(0.0)$ & \\
\hline 1 & $84(80.0)$ & $42(80.8)$ & $42(79.2)$ & \\
\hline 2 & $10(9.5)$ & $0(0.0)$ & 10 (18.9) & \\
\hline 3 & $1(1.0)$ & $0(0.0)$ & $1(1.9)$ & \\
\hline Fibrosis, n (\%) & & & & 0.156 \\
\hline 0 & $42(40.0)$ & $24(46.2)$ & $18(34.0)$ & \\
\hline 1 & $37(35.2)$ & $20(38.5)$ & $17(32.1)$ & \\
\hline 2 & $21(20.0)$ & 7 (13.5) & $14(26.4)$ & \\
\hline 3 & $5(4.8)$ & $1(1.9)$ & $4(7.5)$ & \\
\hline 4 & $0(0.0)$ & $0(0.0)$ & $0(0.0)$ & \\
\hline
\end{tabular}

Continuous variables are presented as mean \pm SD; categorical variables are presented as $\mathrm{n}(\%)$.

$P$ values $<0.05$ are in bold.

AFP, alpha-fetoprotein; ALP, alkaline phosphatase; ALT, alanine aminotransferase; AST, aspartate aminotransferase; BMI, body mass index; BUN, blood urea nitrogen; CK18-M30, cytokeratin-18M30 fragments; GGT, gamma-glutamyl transferase; GP73, Golgi protein 73; HbA1c, hemoglobin A1c; HDL, high-density lipoprotein; HOMA-IR, homeostasis model assessment of insulin resistance; LDL, lowdensity lipoprotein; MPV, mean platelet volume; nALT, normal ALT; NASH, non-alcoholic steatohepatitis; PLT, platelet; RBC, red blood cell; SDPV, SD of platelet volume; WCC, white cell count.

cut-off value of ALT ( $\leq 35 \mathrm{U} / \mathrm{L}$ for men and $\leq 23 \mathrm{U} / \mathrm{L}$ for women, respectively) in defining the nALT status (online supplementary table 3$)$.
Development of a prediction model for diagnosing NASH in patients with persistent nALT

To further differentiate NASH from non-NASH in patients

Table 3 Performance of serum GP73 and CK18-M30 levels in diagnosing NASH in patients with persistent nALT

\begin{tabular}{|c|c|c|c|c|c|c|c|c|}
\hline \multirow[b]{2}{*}{ Tests results } & \multicolumn{2}{|c|}{ Liver pathology results } & \multirow[b]{2}{*}{ Accuracy (\%) } & \multirow[b]{2}{*}{ Se $(\%)$} & \multirow[b]{2}{*}{ Sp (\%) } & \multirow[b]{2}{*}{ PPV } & \multirow[b]{2}{*}{ NPV } & \multirow[b]{2}{*}{ P value* } \\
\hline & NASH & Non-NASH & & & & & & \\
\hline \multicolumn{9}{|l|}{ GP73 } \\
\hline Positive predictive & 41 & 35 & 0.552 & 0.773 & 0.327 & 0.539 & 0.586 & 0.707 \\
\hline Negative predictive & 12 & 17 & & & & & & \\
\hline \multicolumn{9}{|l|}{ CK18-M30 } \\
\hline Positive predictive & 5 & 1 & 0.516 & 0.100 & 0.978 & 0.821 & 0.516 & \\
\hline Negative predictive & 45 & 44 & & & & & & \\
\hline
\end{tabular}

10 missing values in serum CK18-M30.

Diagnostic accuracy was calculated as the sum of true positive and true negative values divided by the total number of observations.

The cut-off value for normal serum ALT was $<40 \mathrm{U} / \mathrm{L}$ for both sexes. Test results above the cut-off value $(53 \mathrm{ng} / \mathrm{mL}$ for GP73 and $200 \mathrm{U} / \mathrm{L}$ for CK18-M30) were considered predictive of $\mathrm{NASH}$.

${ }^{*} \chi^{2}$ test for comparing the diagnostic accuracy of serum GP73 and CK18-M30 levels.

ALT, alanine aminotransferase; CK18-M30, cytokeratin-18 M30 fragments; GP73, Golgi protein 73; nALT, normal ALT; NASH, non-alcoholic steatohepatitis; NPV, negative predictive value; PPV, positive predictive value; Se, sensitivity; Sp, specificity. 
A

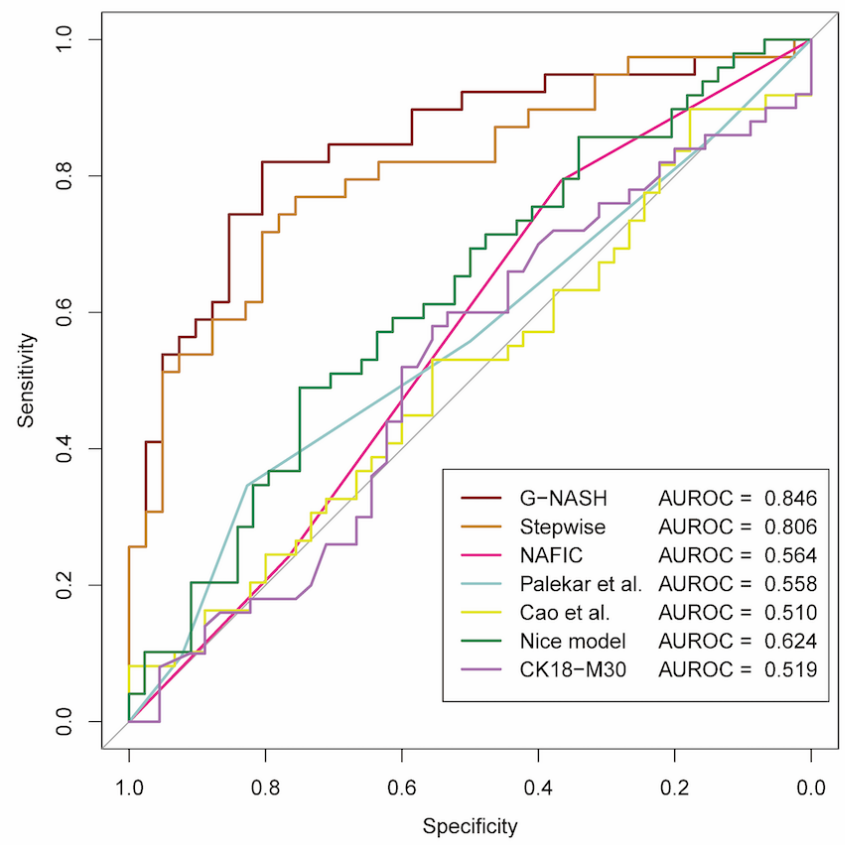

B

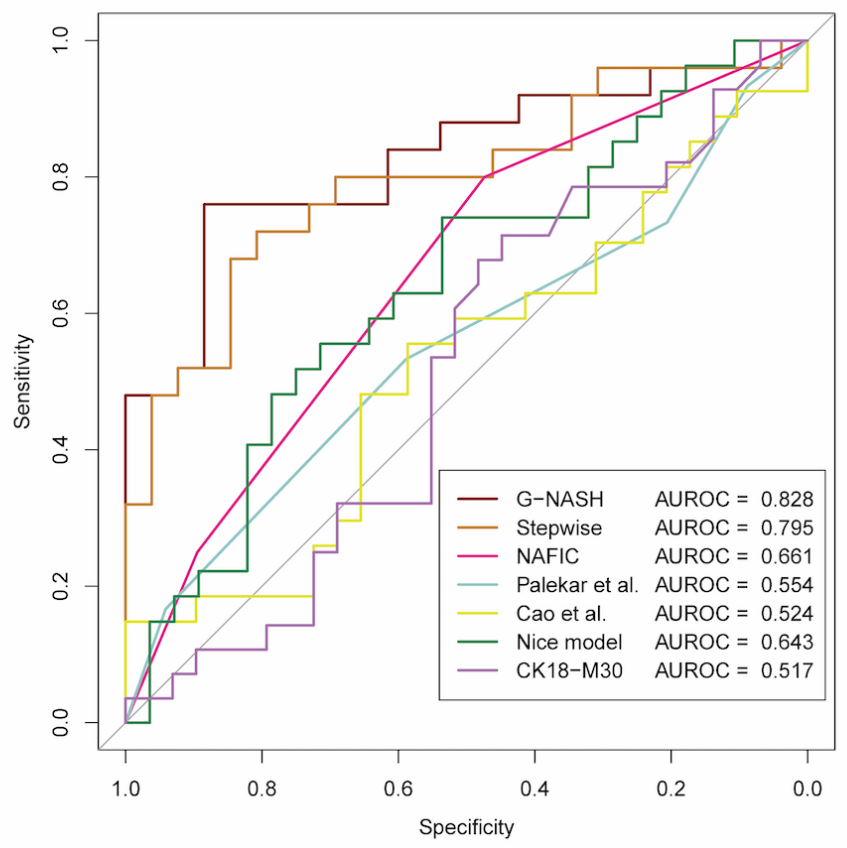

Figure 2 Receiver operating characteristic curves of various non-invasive clinical scoring systems for the prediction of NASH among patients with NAFLD with persistent nALT: (A) using a cut-off of serum ALT levels $<40 \mathrm{U} / L$ for both sexes and (B) using a lower cut-off of serum ALT $\leq 35 \mathrm{U} / \mathrm{L}$ in men $(n=48)$ and $\leq 23 \mathrm{U} / \mathrm{L}$ in women $(n=16)$. The $y$-axis represents the sensitivity in ascending order. The x-axis represents the specificity in descending order. G-NASH is the model constructed by bestsubset regression. Stepwise refers to the logistic regression model constructed by backward stepwise selection. ALT, alanine aminotransferase; AUROC, area under the receiver operating characteristic curve; CK18-M30, cytokeratin-18 M30 fragments; NAFLD, non-alcoholic fatty liver disease; nALT, normal ALT; NASH, non-alcoholic steatohepatitis.

with persistent nALT, we performed both univariable and multivariable logistic regression analyses. Values of GP73, AST, zinc and SD of platelet volume (SDPV) were independently associated with the presence of NASH, and an SLR model was also created (online supplementary table 4): $0.0186 \times \mathrm{GP} 73(\mathrm{ng} / \mathrm{mL})+0.1405 \times \mathrm{AST}$ $(\mathrm{U} / \mathrm{L})+0.1352 \times$ serum zinc $(\mu \mathrm{mol} / \mathrm{L})-0.3969 \times \mathrm{SDPV}$ (fL). The resulting BSR model contained values of serum GP73, zinc, AST, total thyroxine, SDPV and presence of BMI $\geq 30 \mathrm{~kg} / \mathrm{m}^{2}$. Thus, a final BSR model (named G-NASH as it primarily uses GP73 to predict NASH) was calculated as follows: $0.02 \times \mathrm{GP} 73(\mathrm{ng} / \mathrm{mL})+0.123 \times \mathrm{AST}$ $(\mathrm{U} / \mathrm{L})+0.1576 \times$ serum zinc $(\mu \mathrm{mol} / \mathrm{L})+0.0227 \times$ total thyroxine $(\mathrm{nmol} / \mathrm{L})-0.4525 \times \mathrm{SDPV} \quad(\mathrm{fL})+2.0789 \times(\mathrm{BMI}$ $\geq 30 \mathrm{~kg} / \mathrm{m}^{2}$, yes $=1, \mathrm{no}=0$ ). A nomogram is also provided in online supplementary figure 3 to show the possible application of the G-NASH model in clinical practice.

\section{Performance of clinical scoring systems for predicting NASH}

To compare the performance of the G-NASH model with other available clinical scoring systems in identifying NASH, we performed ROC curve comparisons (figure 2). The G-NASH model showed the best AUROC $(0.846,95 \%$ CI 0.757 to 0.934$)$ in diagnosing NASH compared with the SLR model $(0.806,95 \%$ CI 0.708 to 0.903 ) (figure 2A and online supplementary table 1 ). The good diagnostic performance of the G-NASH model was further confirmed even when we used a lower cut-off value of serum ALT levels ( $\leq 35 \mathrm{U} / \mathrm{L}$ for men and $\leq 23 \mathrm{U} / \mathrm{L}$ for women, respectively) (figure 2B). ${ }^{23}{ }^{24}$ The possible reasons for the poorer performances of other available clinical scoring systems in identifying NASH we observed in our study are most likely due to differences in study populations.

Moreover, the clinical value of the G-NASH model was also evaluated by DCA. As shown in online supplementary figure $1 \mathrm{~A}$, decision curves were plotted with $\mathrm{sNB}$ versus $\mathrm{P}_{\mathrm{t}}$ and the CBR in all predictive models and predictors, as well as with the 'treat all' and 'treat none' strategy. By using the G-NASH model and a $\mathrm{P}_{\mathrm{t}}$ higher than 0.22 , almost all of these patients had the highest sNB compared with all other predictive decision models or predictor(s). We also found an $\mathrm{sNB}=0.62$ with $\mathrm{P}_{\mathrm{t}}=0.40$; these data indicate that in patients with persistent nALT, the G-NASH model had $62 \%$ accuracy in identifying NASH and $100 \%$ accuracy in identifying non-NASH.

Online supplementary figure 1B shows the predictive ability of the G-NASH model with different $\mathrm{P}_{\mathrm{t}}$ thresholds in identifying NASH. The solid line denotes the true positive rate and the dotted line denotes the false positive rate. A $\mathrm{P}_{\mathrm{t}}$ of between 0.4 and 0.5 yielded the best test performance and provided the optimal sensitivity and specificity in accurately identifying NASH in patients with persistent nALT. 
Online supplementary figure $1 \mathrm{C}$ shows the potential clinical impact curve of the G-NASH model. The solid line denotes the number of patients identified as NASH by the G-NASH model, given a specific $\mathrm{P}_{\mathrm{t}}$ (high risk), whereas the dotted line denotes the number of patients who truly had NASH at the $\mathrm{P}_{\mathrm{t}}$ (high risk with NASH). When the $\mathrm{P}_{t}$ was $40 \%, 58$ out of 100 patients were identified as NASH, compared with 40 patients who were confirmed with NASH by liver biopsy.

\section{Combined use of non-invasive markers to predict NASH in patients with persistent nALT}

Serum CK18-M30 has better specificity than serum GP73, while GP73 has better sensitivity in diagnosing NASH. Therefore, we devised a combined sequential strategy for identifying patients with persistent nALT who require liver biopsy due to a high probability of NASH. Using the better specificity of CK18-M30, patients would first be assessed by serum CK18-M30 measurement alone. Those patients with serum CK18-M30 higher than the upper normal limit, that is, equal or greater than $200 \mathrm{U} / \mathrm{L}$, were deemed as having NASH, whereas those patients below this CK-18M30 threshold should undergo the G-NASH assessment. Patients with G-NASH score of less than 2 were deemed as having NAFL (simple steatosis), while those with greater than 4 were diagnosed as NASH. Patients with G-NASH score between 2 and 4 ('grey zone') would undergo liver biopsy for staging of NAFLD (online supplementary figure 2). Among the patients assessed with our proposed combined and sequential strategy, 5 out of 70 subjects were directly deemed as having NASH according to serum CK18-M30 measurement alone, with one individual actually having non-alcoholic fatty liver (NAFL), so that the rate of misdiagnosis (Mis) was 20\% (Mis \% (n/N): 20\% (1/5)). The G-NASH model further assessed 65 patients, and 6 of them were deemed as having NAFL and 30 of them as having NASH, with 1 patient (Mis \% (n/N): 16.7\% (1/6)) and 5 patients (Mis \% (n/N): 16.7\% (5/30)) misdiagnosed, respectively. In summary, 7 out of 41 patients were misdiagnosed by our proposed combined and sequential approach without a biopsy, yielding a misdiagnosis rate of $17.1 \%$ (Mis $\%(\mathrm{n} / \mathrm{N}): 17.1 \%(7 / 41))$. By this two-step preselection, $41.4 \%$ (29 out of 70) of our patients with persistent nALT needed biopsy, compared with $92.9 \%$ (65 out of 70) of patients when we used only serum CK18-M30 levels alone (by the standard that patients with serum CK18-M30 levels $<200 \mathrm{U} / \mathrm{L}$ would undergo liver biopsy and those with serum CK18-M30 levels $\geq 200 \mathrm{U} / \mathrm{L}$ were diagnosed as having $\mathrm{NASH}$, without further application of the G-NASH scoring system). Of note, 29 out of 70 patients with persistent nALT still need liver biopsy by our sequential approach, which is a result less than ideal. However, it is also important to note that 41 out of 70 patients may be spared from liver biopsy examination, which is a sizeable improvement especially if we consider the poor specificity and sensibility of currently available non-invasive tests for diagnosing NASH among patients with NAFLD and persistent nALT.

\section{DISCUSSION}

To the best of our knowledge, this is the first study that compared the performance of clinical scoring systems and serum biomarkers for predicting NASH in patients with biopsy-confirmed NAFLD who have persistent nALT levels. In contrast to the few available retrospective studies that have included patients with NAFLD with nALT levels, ${ }^{67}$ our study involves a relatively large cohort of well-characterized patients with biopsy-proven NAFLD, including also a substantial number of patients with persistent nALT levels.

We found that measurement of either serum CK18M30 or GP73 levels alone was not able to accurately identify NASH in the subgroup of patients with NAFLD with persistent nALT levels. However, we found that serum CK18-M30 levels can be useful during an initial screening test, and then sequentially combined with a newly developed G-NASH model that includes measurement of serum GP73 and other clinical/biochemical parameters in its equation. Indeed, our results show that the G-NASH model has good diagnostic accuracy in identifying NASH, and by using this model it should be possible to accurately identify those subjects who require a liver biopsy.

Serum ALT levels are the most widely used laboratory test for assessing and monitoring liver injury. Both in China and in many other countries, serum ALT levels are often used by primary care clinicians for referring patients to gastroenterologists/hepatologists for further investigation. However, the fact that increased serum ALT levels can be present only in a proportion of patients with NAFLD (be it simple steatosis or NASH) necessitates finding alternative noninvasive tests to refine specialist referral pathways. ${ }^{7}$ Previous efforts have been made to discover novel biomarkers or test panels to better distinguish NASH from simple steatosis. Some studies have suggested that measurement of serum CK18-M30 levels may be useful, ${ }^{26}{ }^{27}$ although one study has concluded that CK18-M30 measurement alone lacked sufficient sensitivity for diagnosing NASH. ${ }^{28}$ Similarly, in the present study, we found that a substantial number of patients with persistent nALT (53 out of 105) had NASH on histology, and more importantly serum CK18-M30 levels alone did not have sufficiently high diagnostic accuracy for identifying NASH in this group of patients. Thus, based on these findings, we could make the following inferences: (1) screening for NASH is required in patients with NAFLD who have persistent nALT due to the progressive potential of their liver disease; and (2) serum CK18-M30 measurement alone does not have sufficient sensitivity for detecting the early stages of NASH in this group of patients.

We have also tested the performance of serum GP73 levels in identifying NASH in patients with NAFLD with persistent nALT. A recent study pertaining to hepatocellular carcinoma development showed increased GP73 secretion by hepatocyte endoplasmic reticulum (ER) stress. ${ }^{29}$ In view of the recognized relevance of ER stress in NAFLD pathogenesis, it is also possible to speculate that upregulation of gene expression and increased serum levels of GP73 may also occur in NASH..$^{30}$ Additionally, 
it has been reported that interferon gamma (IFN- $\gamma$ ) was responsible for increased expression of GP73 in hepatocytes, ${ }^{31}$ and elevated IFN- $\gamma$-secreting T helper cell 17 infiltration was also found in the hepatic tissue relative to peripheral blood mononuclear cells. ${ }^{32}$ Collectively, these findings might, at least in part, explain the significant association we observed between serum GP73 levels and the presence of NASH.

Increased platelet volume may occur with inflammatory states, ${ }^{33}$ and alterations in serum zinc ${ }^{34}$ and thyroxine levels ${ }^{35}$ have been noted to be associated with NAFLD. Zinc is mostly found in skeletal muscle rather than the liver, and fluctuation of serum zinc levels may occur with sarcopenia, ${ }^{36}$ which has been noted with NAFLD. ${ }^{37}$ Therefore, since NASH is a proinflammatory state, there is emerging evidence supporting the inclusion of SDPV, serum zinc and thyroxine levels in the prediction model.

In clinical practice, the use of liver biopsy is limited mostly due to its poor acceptability to patients, high costs, and high risk to benefit ratio. Among patients with NAFLD with nALT levels, this issue is even more pertinent, as clinicians are more inclined to investigate only patients with abnALT levels. Since it is known that most of the available non-invasive tests/methods perform poorly in diagnosing NASH, better non-invasive tests are increasingly needed, especially for screening the subgroup of patients with NAFLD with nALT levels, who may be otherwise misdiagnosed as having less harmful disease by non-specialists. In this regard, serum CK18M30 levels and other non-invasive clinical scoring systems have been previously investigated for their ability to accurately identify NASH in patients with NAFLD and abnALT. ${ }^{27}{ }^{38}$ However, these non-invasive clinical scoring systems are complex combinations that include 'omics' methodology, which is usually not available in clinical practice.

To resolve this conundrum, we have proposed a sequential non-invasive approach by using serum CK18-M30 measurement first and then applying the G-NASH model. In our cohort of patients with biopsy-confirmed NAFLD, serum GP73 had better sensitivity than serum CK18-M30 alone for the diagnosis of NASH among those patients with persistent nALT, thus suggesting that serum GP73 might be superior to CK18-M30 alone in diagnosing the early stages of NASH. However, it is noteworthy that increased serum CK18-M30 might reflect prominent hepatic necroinflammation, which typically occurs in NASH, but not NAFL. ${ }^{39}$ Therefore, we propose that serum CK18-M30 measurement alone could be used first to screen out apparent cases of NASH, with subsequent use of the G-NASH model (which includes serum GP73 concentration along with BMI and other laboratory parameters) for accurately differentiating NASH from simple steatosis in the remaining patients. Notably, this proposed combined and sequential approach for non-invasively identifying NASH was effective in reducing unnecessary liver biopsies with an acceptable misdiagnosis rate.
Among the major limitations of our single-center, observational study, we need to mention the lack of an external validation cohort to test our proposed strategy of using a sequential combination of serum CK18-M30 and the G-NASH model for non-invasively identifying patients with NASH among NAFLD with persistent nALT. In addition, serum CK18-M30 and GP73 levels are not measured in routine clinical practice. Our study also included a relatively small number of patients $(n=53)$ with NASH who had persistent nALT. With this small number of subjects we were unable to stratify the analyses by sex in order to test the prediction model in men and women, separately. Patients included in our study were of Chinese Han ethnicity, precluding extrapolation of these results to other ethnic groups. Thus, our results need to be verified in larger studies that also include other ethnic groups.

In conclusion, our results show that $~ 50 \%$ of patients with NAFLD with persistent nALT levels have NASH, and the sequential combination of serum CK18-M30 measurement and the G-NASH model can be used to identify NASH. Not only does this approach accurately identify patients with NASH, but importantly it also reduces the number of patients who may be subjected to an unnecessary liver biopsy.

\section{Author affiliations}

${ }^{1}$ NAFLD Research Center, Department of Hepatology, The First Affiliated Hospital of Wenzhou Medical University, Wenzhou, China

${ }^{2}$ Department of Endocrinology, The First Affiliated Hospital of Wenzhou Medical University, Wenzhou, China

${ }^{3}$ Department of Laboratory Medicine, The First Affiliated Hospital of Wenzhou Medical University, Wenzhou, China

${ }^{4}$ Department of Gastroenterology, The First Affiliated Hospital of Wenzhou Medical University, Wenzhou, China

${ }^{5}$ Division of Endocrinology and Metabolism, University of Verona, 0spedale Civile Maggiore, Verona, Italy

${ }^{6}$ Southampton National Institute for Health Research Biomedical Research Centre, University Hospital Southampton, Southampton General Hospital, Southampton, UK ${ }^{7}$ Institute of Hepatology, Wenzhou Medical University, Wenzhou, China

${ }^{8}$ Key Laboratory of Diagnosis and Treatment for The Development of Chronic Liver Disease in Zhejiang Province, Wenzhou, China

${ }^{9}$ Department of Microbiology and Infectious Disease Center, School of Basic Medicine, Peking University Health Science Center, Beijing, China

Acknowledgements The authors thank Professor Ji-Min Liu, a pathologist from McMaster University, Canada, who conducted quality control of liver pathology data. The authors also thank Hotgen Biotech (Beijing, China) and Herui Biomed Company (Suzhou, China) for providing ELISA kits. This work is a part of the PERSONS study.

Contributors Study concept and design: Y-PC, FL and M-HZ. Acquisition of data: W-YL, X-YP, H-LM, P-WZ and X-XW. Pathology analysis: X-DW. Analysis and interpretation of data: KIZ. Drafting of the manuscript: KIZ. Critical revision of the manuscript for important intellectual content: GT and CB. Study supervision: FL and $\mathrm{M}-\mathrm{HZ}$. All authors contributed to the manuscript for important intellectual content and approved the submission.

Funding This work was supported by grants from the National Natural Science Foundation of China (81500665), High Level Creative Talents from Department of Public Health in Zhejiang Province, and Project of New Century 551 Talent Nurturing in Wenzhou. GT is supported in part by grants from the University School of Medicine of Verona, Verona, Italy. CB is supported in part by the Southampton NIHR Biomedical Research Centre (IS-BRC-20004), UK.

Competing interests None declared.

Patient consent for publication Not required. 
Ethics approval The research protocol of this study was approved by the ethics committee of The First Affiliated Hospital of Wenzhou Medical University. All procedures followed were in accordance with the local ethical standards of the responsible committee on human experimentation and with the Helsinki Declaration of 1975, as revised in 2008. Informed consent was obtained from all patients for being included in the study.

Provenance and peer review Not commissioned; externally peer reviewed.

Data availability statement Data are available upon reasonable request.

Open access This is an open access article distributed in accordance with the Creative Commons Attribution Non Commercial (CC BY-NC 4.0) license, which permits others to distribute, remix, adapt, build upon this work non-commercially, and license their derivative works on different terms, provided the original work is properly cited, appropriate credit is given, any changes made indicated, and the use is non-commercial. See: http://creativecommons.org/licenses/by-nc/4.0/.

\section{ORCID iDs}

Kenneth I Zheng http://orcid.org/0000-0002-1726-9298

Giovanni Targher http://orcid.org/0000-0002-4325-3900

Ming-Hua Zheng http://orcid.org/0000-0003-4984-2631

\section{REFERENCES}

1 Younossi ZM, Blissett D, Blissett R, et al. The economic and clinical burden of nonalcoholic fatty liver disease in the United States and Europe. Hepatology 2016;64:1577-86.

2 Chalasani N, Younossi Z, Lavine JE, et al. The diagnosis and management of nonalcoholic fatty liver disease: practice guidance from the American association for the study of liver diseases. Hepatology 2018;67:328-57.

3 Committee of Hepatology, Chinese Research Hospital Association, Fatty Liver Expert Committee, Chinese Medical Doctor Association, National Workshop on Fatty Liver and Alcoholic Liver Disease, Chinese Society of Hepatology, et al.. [Expert recommendations on standardized diagnosis and treatment for fatty liver disease in China (2019 revised edition)]. Zhonghua Gan Zang Bing Za Zhi 2019;27:748-53.

4 Byrne CD, Patel J, Scorletti E, et al. Tests for diagnosing and monitoring non-alcoholic fatty liver disease in adults. BMJ 2018;362:k2734.

5 Health USDo, Control HSCfD, Statistics PNCfH. National Health and Nutrition Examination Survey III, 1988-1994. In: Inter-university Consortium for Political and Social Research [distributor], 1998.

6 Gawrieh S, Wilson LA, Cummings OW, et al. Histologic findings of advanced fibrosis and cirrhosis in patients with nonalcoholic fatty liver disease who have normal aminotransferase levels. Am J Gastroenterol 2019;114:1626-35

7 Wong VW-S, Wong GL-H, Tsang SW-C, et al. Metabolic and histological features of non-alcoholic fatty liver disease patients with different serum alanine aminotransferase levels. Aliment Pharmacol Ther 2009;29:387-96.

8 Yilmaz Y, Kurt R, Kalayci C. Apoptosis in nonalcoholic steatohepatitis with normal aminotransferase values: zooming in on cytokeratin 18 fragments. Biomark Med 2010;4:743-5.

9 Yao M, Wang L, Leung PSC, et al. The clinical significance of GP73 in immunologically mediated chronic liver diseases: experimental data and literature review. Clin Rev Allergy Immunol 2018;54:282-94.

10 Yao M, Wang L, You H, et al. Serum GP73 combined AST and GGT reflects moderate to severe liver inflammation in chronic hepatitis $\mathrm{B}$. Clin Chim Acta 2019;493:92-7.

11 Lu FM, Zhang Y. [Diagnostic application of serum GP73 and the relevant mechanism in the diagnosis of liver cirrhosis]. Zhonghua Gan Zang Bing Za Zhi 2018;26:321-4.

12 Zhou Y-J, Ye F-Z, Li Y-Y, et al. Individualized risk prediction of significant fibrosis in non-alcoholic fatty liver disease using a novel nomogram. United European Gastroenterol J 2019;7:1124-34.

13 Liu W-Y, Zheng KI, Pan X-Y, et al. Effect of PNPLA3 polymorphism on diagnostic performance of various noninvasive markers for diagnosing and staging nonalcoholic fatty liver disease. $J$ Gastroenterol Hepatol 2019. doi:10.1111/jgh.14894. [Epub ahead of print: 01 Nov 2019].

14 Matthews DR, Hosker JP, Rudenski AS, et al. Homeostasis model assessment: insulin resistance and ?-cell function from fasting plasma glucose and insulin concentrations in man. Diabetologia 1985;28:412-9.

15 Leung JC-F, Loong TC-W, Wei JL, et al. Histological severity and clinical outcomes of nonalcoholic fatty liver disease in nonobese patients. Hepatology 2017;65:54-64.
16 Anty R, lannelli A, Patouraux S, et al. A new composite model including metabolic syndrome, alanine aminotransferase and cytokeratin-18 for the diagnosis of non-alcoholic steatohepatitis in morbidly obese patients. Aliment Pharmacol Ther 2010;32:1315-22.

17 Cao W, Zhao C, Shen C, et al. Cytokeratin 18, alanine aminotransferase, platelets and triglycerides predict the presence of nonalcoholic steatohepatitis. PLoS One 2013;8:e82092.

18 Palekar NA, Naus R, Larson SP, et al. Clinical model for distinguishing nonalcoholic steatohepatitis from simple steatosis in patients with nonalcoholic fatty liver disease. Liver Int 2006;26:151-6.

19 Sumida $\mathrm{Y}$, Yoneda M, Hyogo $\mathrm{H}$, et al. A simple clinical scoring system using ferritin, fasting insulin, and type IV collagen $7 \mathrm{~S}$ for predicting steatohepatitis in nonalcoholic fatty liver disease. $J$ Gastroenterol 2011;46:257-68.

20 Ye F-Z, Liu W-Y, Zheng KI, et al. Homeostatic model assessment of insulin resistance closely related to lobular inflammation in nonalcoholic fatty liver disease. Eur J Gastroenterol Hepatol 2020;32:80-6.

21 Kleiner DE, Brunt EM, Van Natta M, et al. Design and validation of a histological scoring system for nonalcoholic fatty liver disease. Hepatology 2005;41:1313-21.

22 Brunt EM, Janney CG, Bisceglie AM, et al. Nonalcoholic steatohepatitis: a proposal for grading and staging the histological lesions. Am J Gastroenterol 1999;94:2467-74.

23 Kwo PY, Cohen SM, Lim JK. Acg clinical guideline: evaluation of abnormal liver chemistries. Am J Gastroenterol 2017;112:18-35.

24 Zheng M-H, Shi K-Q, Fan Y-C, et al. Upper limits of normal for serum alanine aminotransferase levels in Chinese Han population. PLOS One 2012;7:e43736.

25 Van Calster B, Wynants L, Verbeek JFM, et al. Reporting and interpreting decision curve analysis: a guide for Investigators. Eur Urol 2018;74:796-804

26 Tada T, Kumada T, Toyoda $\mathrm{H}$, et al. New scoring system combining the FIB-4 index and cytokeratin-18 fragments for predicting steatohepatitis and liver fibrosis in patients with nonalcoholic fatty liver disease. Biomarkers 2018:23:328-34.

27 Miyasato M, Murase-Mishiba Y, Bessho M, et al. The cytokeratin-18 fragment level as a biomarker of nonalcoholic fatty liver disease in patients with type 2 diabetes mellitus. Clinica Chimica Acta 2014;433:184-9.

28 Cusi K, Chang Z, Harrison S, et al. Limited value of plasma cytokeratin-18 as a biomarker for NASH and fibrosis in patients with non-alcoholic fatty liver disease. J Hepatol 2014;60:167-74.

29 Wei C, Yang X, Liu N, et al. Tumor microenvironment regulation by the endoplasmic reticulum stress transmission mediator Golgi protein 73 in mice. Hepatology 2019:70:851-70.

30 Lebeaupin C, Vallée D, Hazari Y, et al. Endoplasmic reticulum stress signalling and the pathogenesis of non-alcoholic fatty liver disease. $J$ Hepatol 2018:69:927-47.

31 Kladney RD, Cui X, Bulla GA, et al. Expression of GP73, a resident Golgi membrane protein, in viral and nonviral liver disease. Hepatology 2002;35:1431-40.

32 Rau M, Schilling A-K, Meertens J, et al. Progression from nonalcoholic fatty liver to nonalcoholic steatohepatitis is marked by a higher frequency of Th17 cells in the liver and an increased Th17/ Resting regulatory T cell ratio in peripheral blood and in the liver. $J$ Immunol 2016;196:97-105.

33 Korniluk A, Koper-Lenkiewicz OM, Kamińska J, et al. Mean platelet volume (MPV): new perspectives for an old marker in the course and prognosis of inflammatory conditions. Mediators Inflamm 2019;2019:9213074

34 Ito T, Ishigami M, Ishizu $\mathrm{Y}$, et al. Correlation of serum zinc levels with pathological and laboratory findings in patients with nonalcoholic fatty liver disease. Eur J Gastroenterol Hepatol 2019:1

35 Lonardo A, Mantovani A, Lugari S, et al. Nafld in some common endocrine diseases: prevalence, pathophysiology, and principles of diagnosis and management. Int J Mol Sci 2019;20:2841.

36 Hanai T, Shiraki M, Nishimura K, et al. Sarcopenia impairs prognosis of patients with liver cirrhosis. Nutrition 2015;31:193-9.

37 De Fré CH, De Fré MA, Kwanten WJ, et al. Sarcopenia in patients with non-alcoholic fatty liver disease: is it a clinically significant entity? Obesity Reviews 2019;20:353-63.

38 Feldstein AE, Wieckowska A, Lopez AR, et al. Cytokeratin-18 fragment levels as noninvasive biomarkers for nonalcoholic steatohepatitis: a multicenter validation study. Hepatology 2009;50:1072-8.

39 Tsutsui M, Tanaka N, Kawakubo M, et al. Serum fragmented cytokeratin 18 levels reflect the histologic activity score of nonalcoholic fatty liver disease more accurately than serum alanine aminotransferase levels. J Clin Gastroenterol 2010;44:1-447. 